hep-th/9606082

PUPT-1627

ITEP-TH-18/96

YCTP-P10-96

\title{
Chiral Lagrangians, Anomalies, Supersymmetry, and Holomorphy
}

\author{
Andrei Losev ${ }^{1}$, Gregory Moore ${ }^{2}$, Nikita Nekrasov ${ }^{3}$, and Samson Shatashvili ${ }^{4 *}$ \\ 1,3 Institute of Theoretical and Experimental Physics, 117259, Moscow, Russia \\ 3 Department of Physics, Princeton University, Princeton NJ 08544 \\ 1,2,4 Dept. of Physics, Yale University, New Haven, CT 06520, Box 208120 \\ losev@waldzell.physics.yale.edu \\ moore@castalia.physics.yale.edu \\ nikita@puhep1.princeton.edu \\ samson@euler.physics.yale.edu
}

We investigate higher-dimensional analogues of the $b c$ systems of 2D RCFT. When coupled to gauge fields and Beltrami differentials defining integrable holomorphic structures the $b c$ partition functions can be explicitly evaluated using anomalies and holomorphy. The resulting induced actions generalize the chiral algebras of $2 \mathrm{D}$ RCFT to $2 n$ dimensions. Moreover, $b c$ systems in four and six dimensions are closely related to supersymmetric matter. In particular, we show that $d=4, \mathcal{N}=2$ hypermultiplets induce a theory of selfdual Yang-Mills fields coupled to self-dual gravity. In this way the $b c$ systems fermionize both the algebraic sector of the $W Z W_{4}$ theory, as defined by Losev et. al., and the classical open $\mathcal{N}_{w s}=2$ string.

\footnotetext{
* On leave of absence from St. Petersburg Steklov Mathematical Institute, St. Petersburg, Russia.
} 
June 11, 1996 


\section{Introduction}

This paper continues an investigation into the application and generalization of twodimensional conformal field theory to analogous higher dimensional theories begun in [1] [2] [3]. We will show that the theories studied in [1][2] [3] are closely related to supersymmetric models. In particular, we consider actions for gauge and gravitational fields induced by integrating over chiral multiplets and hypermultiplets in four and six dimensions. For certain background fields the induced action can be written explicitly as a "sigma model Lagrangian" in a class of Lagrangians we name chiral cocycle Lagrangians. The chiral cocycle Lagrangians are naturally defined for all $2 n$-dimensional spacetimes with a complex structure. Moreover they are derivable from characteristic classes. The chiral cocycle theories generalize both the $W Z W_{2}$ theory of two dimensions [4] (including its gravitational generalization [5] ) and its four dimensional counterpart investigated in [6] [1]. These results are in close analogy to the results of Polyakov and Wiegmann and Polyakov [7] [8] [5] in two-dimensions.

To motivate the theories let us begin by recalling the important notion of twodimensional $b c$ systems [9] [10]. These are defined by choosing a complex structure on a surface $X_{2}$ and introducing anticommuting $(1,0)$-form and $(0,0)$-form fields $b_{m, z}(z, \bar{z})$, $c^{m}(z, \bar{z})$, where $m=1, \ldots, r$ is a flavor index. These fields may be coupled to the $(0,1)$ part of a gauge field, $\bar{a}$, and a Beltrami differential $\bar{\mu}_{\bar{z}}{ }^{z}(z, \bar{z})$, through the action:

$$
\int b(\bar{\partial}+\bar{\mu} \cdot \partial+\bar{a}) c
$$

One can develop all of (rational) conformal field theory from the study of the partition function

$$
\mathcal{Z}_{c h}(\bar{a} ; \bar{\mu})=\int[d b d c] e^{\int b(\bar{\partial}+\bar{\mu} \cdot \partial+\bar{a}) c}=\left\langle e^{\int \bar{\mu} T+\bar{a} J}\right\rangle
$$

The second equation emphasizes the interpretation of $\mathcal{Z}_{c h}$ as the generating function of current correlators. On the other hand, in two dimensions, $\bar{a}$ is (locally) holomorphically pure gauge, e.g., $\bar{a}=g^{-1} \bar{\partial} g$ for a $G L(r, \mathbb{C})$ gauge transformation $g$. Using the analytically continued formula for the anomaly one easily evaluates (1.2), discovering current algebra from the Lie algebra cocycle and the $W Z W_{2}$ action from the group cocycle. A similar procedure including $\bar{\mu}$ and the metric leads to the gravitational $W Z W$ theory, the diffeomorphism anomaly, the Virasoro algebra, and Liouville theory.

In this paper we indicate how the above argument generalizes to higher-dimensional theories. We will define a theory analogous to (1.2) in $2 n$ dimensions. For holomorphically 
trivial fields $\bar{a}, \bar{\mu}$ we will use the anomaly to evaluate $\mathcal{Z}_{c h}(\bar{a}, \bar{\mu})$ exactly in terms of a group cocycle.

We generalize (1.1) to higher dimensions simply by writing (11.1) where $c$ is now a $(0, j)$ form on an $n$-complex dimensional manifold $X_{2 n}$ with values in a vector bundle $E \rightarrow X_{2 n}$, $b$ is an $(n, n-1-j)$-form valued in the dual bundle $E^{*}, \bar{a}$ is the $(0,1)$ part of a connection on $E$ and $\bar{\mu}$ is a Beltrami differential. When $n$ is larger than one an essential new element emerges: the number of degrees of freedom in $b, c$ do not balance, and one must introduce further fields to make sense of (1.2). If the background fields satisfy:

$$
(\bar{\partial}+\bar{\mu} \cdot \partial+\bar{a})^{2}=\left(\bar{\partial}_{\bar{\mu}, \bar{a}}\right)^{2}=0
$$

then there is a very natural way to introduce these extra fields: (11.3) implies that (1.1) has a gauge invariance $Y: c \rightarrow c+\bar{\partial}_{\bar{\mu}, \bar{a}} \epsilon$. Gauge-fixing the $Y$-symmetry then leads unambiguously to the higher-dimensional $b c$ theories. We describe this in detail in section two. The gauge-fixed Lagrangian has a BRST symmetry $Q$. It turns out that, on a Kähler manifold the $b c$ system is nothing but a Weyl fermion (together with a collection of bosons) coupled to a gauge field $A=(a, \bar{a})$ with both $(1,0)$ and $(0,1)$ pieces. The $(1,0)$ piece, $a$, enters through gauge fixing. In fact, as we show in section three, in four and six dimensions this theory of bosons and fermions is simply supersymmetric matter, twisted using a Kahler structure as in [11] [12] [13].

The key equation (1.3) has a simple geometric meaning. The equation for $\bar{\mu}$ is the Kodaira-Spencer equation, so the Beltrami differential $\bar{\mu}$ defines an integrable deformation of complex structure, while $\bar{a}$ defines a holomorphic structure on the vector bundle $E$ in this complex structure. The equation (1.3) is solved - locally - by writing the backgrounds as "holomorphically pure gauge"

$$
\begin{aligned}
& \bar{a}=g^{-1}(\bar{\partial}+\bar{\mu} \cdot \partial) g \\
& \bar{\mu}=\bar{\mu}[f]=(\bar{\partial} f)(\partial f)^{-1}
\end{aligned}
$$

where $g(z, \bar{z})$ is a $G L(r, \mathbb{C})$ gauge transformation, $(z, \bar{z}) \rightarrow(f(z, \bar{z}), \bar{z})$ is a chiral diffeomorphism, and $(\partial f)$ is a matrix of derivatives. Of course, in general there are obstructions to writing (1.4) globally because of the existence of moduli. However, the dependence on the underlying groups is of primary importance and is hence the focus of this paper. Thus, in this paper we ignore moduli and zeromodes and focus on the $g$ and $f$ dependence. The very interesting and important problem of dependence on moduli is a subject to which we hope to return. 
In section four we address the definition of the chiral partition function (1.2), and in section five we evaluate it. In direct analogy to the two-dimensional case the partition function (1.2) can be evaluated exactly for the class of backgrounds (1.3). The essential argument for this is extremely simple and proceeds as follows. Since we have a Weyl fermion the chiral partition function must have an anomalous variation under unitary gauge transformations of $A$. In an appropriate regularization (which we will refer to as the "standard regularization")

$$
Z_{c h, s}\left(A^{u}\right)=e^{i \alpha_{s}(A, u)} Z_{c h, s}(A)
$$

where $\alpha_{s}(A, u)$ is the standard anomaly functional derived from descent [14] [15] [16]. 1 1 On the other hand, if $\mathcal{Z}_{c h}$ can be defined maintaining $Q$-invariance then it must be a function of $\bar{a}$ only, that is, it must be $a$-independent. (More generally it must be independent of gauge fixing):

$$
\mathcal{Z}_{c h}(A)=\mathcal{Z}_{c h}(\bar{a})
$$

A glance at the standard formula for $\alpha_{s}$ (see, e.g., eq. (5.2) below) shows that $Z_{c h, s}$ cannot be holomorphic, hence the standard regularization does not preserve $Q$-symmetry. The situation is redeemed by the addition of a local counterterm, or, what is the same, by a change of regularization. In more algebraic langauge, a change of regularization corresponds to a change of the anomalous group 1-cocycle $\alpha_{s}$ by a coboundary $\alpha_{s} \rightarrow \alpha=$ $\alpha_{s}+\delta \gamma$. The absence of a $Y$-anomaly implies the preservation of $Q$-invariance. A necessary condition for $Q$ invariance is the existence of a local functional $\gamma(a, \bar{a})$ such that the group cocycle

$$
\alpha_{h}(\bar{a}, u)=\alpha_{s}(A, u)+\delta \gamma(a, \bar{a}, u)
$$

is $a$-independent. In section 5.1 below we show that such a $\gamma$ exists. After analytic continuation of (1.5) in $u$, we are in a position to gauge away $\bar{a}=g^{-1} \bar{\partial} g$ from $\mathcal{Z}_{c h}$. The result is the chiral cocycle Lagrangian: $\Gamma[g]=i \alpha_{h}(\bar{a}=0, u=g)$ where $g$ is in the complexified gauge group. It is possible to write explicit formulae for $\alpha_{h}$. The Lie algebraic cocycle corresponding to (1.7) is very simply written:

$$
C(\epsilon, \bar{a})=\int_{X_{2 n}} \operatorname{Tr} \epsilon(\partial \bar{a})^{n}
$$

1 In more algebraic language, $\alpha_{s}(A, u)$ is a "1-cocycle taking values in functions of connections." It satisfies $\delta \alpha_{s}=0$ where $(\delta f)(A, g)=f\left(A^{g}\right)-f(A)$ for 0-cochains, $(\delta f)\left(A, g_{1}, g_{2}\right)=f\left(A^{g_{1}}, g_{2}\right)-$ $f\left(A, g_{1} g_{2}\right)+f\left(A, g_{1}\right)$ for 1-cochains, etc. 
and the corresponding chiral cocycle Lagrangian (in four dimensions) is:

$$
\log \mathcal{Z}_{c h}[\bar{a}]=\Gamma[g]=\int_{X_{4}} \operatorname{Tr}\left[2(\ell \bar{\ell}-\bar{\ell} \ell) \bar{\partial} \ell+(\bar{\ell} \ell)^{2}\right]+\frac{2}{5} \int_{X_{4} \times I} \operatorname{Tr}\left(g^{-1} d g\right)^{5}
$$

where $\ell=g^{-1} \partial g, \bar{\ell}=g^{-1} \bar{\partial} g$. As we show in section seven, $\Gamma[g]$ can be obtained directly from a holomorphic version of the descent procedure [14] [15] [16] and has the form

$$
\Gamma[g]=\int_{X_{2 n} \times D} \frac{d w}{w} \wedge \operatorname{Tr} \bar{\ell}(\partial \bar{\ell})^{n}
$$

where $D$ is a disk and we extend $g$ so that $g=1$ on the boundary of $g$.

Studying the $\bar{\mu}$-dependence of (1.2) in a similar spirit leads to analogous formulae for group cocycles for the group of chiral diffeomorphisms. Parametrizing $\bar{\mu}$ as in (1.4) we find in section 8 that $\log \mathcal{Z}_{c h}[\bar{\mu}]=\Gamma\left[J(F)^{-1}\right]$ where $F$ is an inverse to $f$ and $J(F)$ is the $G L(n, \mathbb{C})$ matrix $\partial_{i} F^{j}$, i.e. the chiral Jacobian. The Lie algebra cocycle analogous to (1.8) is simply:

$$
C(v, \bar{\mu})=\int_{X_{2 n}} \operatorname{Tr}\left[(\partial v)\left(\mathcal{R}_{\bar{\mu}}\right)^{n}\right]
$$

where we define a two-form $\mathcal{R}_{\bar{\mu}}$ with values in $g l(n, \mathbb{C})$ by

$$
\left(\mathcal{R}_{\bar{\mu}}\right)_{m}^{p} \equiv d z^{j} d \bar{z}^{\bar{k}}\left(\partial_{j} \partial_{m} \bar{\mu}_{\bar{k}}^{p}\right)
$$

and the trace is in any representation. Given (11.2), (1.11) generalizes the Virasoro anomaly to higher dimensions.

There are some noteworthy implications of the above simple argument. First, the principle of holomorphy determines the kinetic term in the induced "pion Lagrangian." Usually such kinetic terms (and their higher-derivative corrections) are regarded as coboundaries which definitely affect physics but are beyond the reach of the method of descent. Evidently, a principle analogous to our holomorphy principle in the context of QCD would have profound consequences $\mathrm{a}$ Second, quantization of free field theory is in principle straightforward, so we have provided a construction of higher dimensional counterparts of chiral algebras (in the sense of CFT), together with a realization of these algebras generalizing two-dimensional fermionization. 3

2 There are other possible applications to QCD. See, for example, 17] 18.

3 In the special case that $X_{4}$ is a product of Riemann surfaces on can define "projected algebras" which are exactly the chiral algebras of $2 \mathrm{~d}$ CFT. These were first investigated in [11] and further studied in [1]. Related results on higher-dimensional fermionization are described in [19]. 
In section nine we consider the induced action for a four-dimensional $b c$ system in a special representation defined by an $\mathcal{N}=2$ hypermultiplet. In this representation the action (1.9) includes the $W Z W_{4}$ Lagrangian [1]. (Indeed this was the starting point for the present investigation.) The induced theory is also closely related to the classical target space theory of an open string theory with gauged $\mathcal{N}_{w s}=2$ supersymmetry. In particular the Plebanski equation and its modification in the presence of gauge fields naturally emerges from the chiral cocycle Lagrangian. Our results suggest many new questions and interesting avenues of research. Some of these are indicated in the conclusions.

\section{Lagrangians for $b c$ systems in $2 n$ dimensions}

In this section we define more precisely the Lagrangians of the higher-dimensional analogs of the $2 \mathrm{~d} b c$ systems of [9] [10].

\section{1. bc systems in 4 dimensions}

Let $E \rightarrow X$ be a complex rank $r$ hermitian vector bundle over a complex surface $X$. We assume that $E$ is equipped with a $G L(r, \mathbb{C})$ connection $(a, \bar{a})$ so that the $(0,1)$ piece $\bar{\partial}_{\bar{a}}$ defines a holomorphic structure on $E: \bar{\partial}_{\bar{a}}^{2}=0$. Note that we do not assume $F^{2,0}=0$.

The most obvious generalization of bc systems is defined by introducing the fermionic fields

$$
\begin{aligned}
& b \in \Omega^{2,0}\left(X, E^{*}\right) \\
& c \in \Omega^{0,1}(X, E)
\end{aligned}
$$

We try the action: 4

$$
S \stackrel{?}{=} \int_{X}\left\langle b, \bar{\partial}_{\bar{a}} c\right\rangle
$$

This theory does not makes sense: the operator $\bar{\partial}_{\bar{a}}$ is not nearly Fredholm. Indeed, there is a large gauge symmetry:

$$
c \rightarrow c+\bar{\partial}_{\bar{a}} \epsilon
$$

which we refer to as $Y$-symmetry. We now fix it using the Faddeev-Popov procedure. Introduce a ghost $\phi$ and an antighost multiplet $(e, \bar{\phi})$ :

$$
\begin{gathered}
\phi \in \Omega^{0,0}(E) \\
e, \bar{\phi} \in \Omega^{0,0}\left(E^{*}\right)
\end{gathered}
$$

4 The symbol $\langle\cdot, \cdot\rangle$ denotes a bilinear, dual pairing, while $(\cdot, \cdot)$ will denote a Hilbert space inner product, antilinear in the first slot. 
(fields with Greek letters commute, Latin letters anticommute.) We have a BRST Qsymmetry:

$$
\begin{array}{rlrl}
Q: c \rightarrow c+\bar{\partial}_{\bar{a}} \phi & & \phi \rightarrow 0 \\
Q: \bar{\phi} \rightarrow e & e \rightarrow 0
\end{array}
$$

We introduce the gauge-fixing term via the gauge fermion:

$$
\Psi=-i \int_{X} \omega\left\langle\partial_{a} \bar{\phi}, c\right\rangle
$$

where $\omega$ is a positive $(1,1)$ form. For example we can take it to be the imaginary part of an hermitian metric on $X$.

The action of the gauge-fixed chiral bc system is

$$
\begin{aligned}
S_{b c} & =\int_{X}\left\langle b, \bar{\partial}_{\bar{a}} c\right\rangle+\{Q, \Psi\} \\
& =\int_{X}\left\langle b, \bar{\partial}_{\bar{a}} c\right\rangle+\omega\left\langle\partial_{a} e, c\right\rangle+\omega\left\langle\partial_{a} \bar{\phi}, \bar{\partial}_{\bar{a}} \phi\right\rangle
\end{aligned}
$$

The equations of motion are:

$$
\begin{aligned}
\bar{\partial}_{\bar{a}} c=\partial_{a}(\omega c) & =0 \\
\omega \partial_{a} e-\bar{\partial}_{\bar{a}} b & =0 \\
\partial_{a} \omega \bar{\partial}_{\bar{a}} \phi & =0 \\
\bar{\partial}_{\bar{a}} \omega \partial_{a} \bar{\phi} & =0
\end{aligned}
$$

The explicit coupling to the gauge fields is:

$$
-\int \operatorname{Tr}(\bar{a} J+a \bar{J})
$$

where

$$
\begin{aligned}
& J=c \otimes b-\omega \phi \otimes \partial_{a} \bar{\phi} \in \Omega^{2,1}(E n d E) \\
& \bar{J}=\omega c \otimes e+\omega \bar{\partial} \phi \otimes \bar{\phi} \in \Omega^{1,2}(E n d E)
\end{aligned}
$$

In particular using the equations of motion we have:

$$
\{Q, J\}=-\bar{\partial}_{\bar{a}}(b \phi)
$$

and

$$
\bar{\partial}_{\bar{a}} J=\left\{Q, \omega \partial_{a} \bar{\phi} c\right\}
$$


Of crucial importance in this paper is the fact, following immediately from (2.7), that the coupling to $a$ is $Q$-exact:

$$
\frac{\delta}{\delta a} S=\bar{J}+\omega \bar{a} \phi \otimes \bar{\phi}=\{Q, \omega c \bar{\phi}\}
$$

and hence the path integral must be $a$-independent. This is simply an example of the independence of gauge slice in Faddeev-Popov gauge fixing. More generally, one should be able to obtain equivalent results using different gauge fermions in (2.7). The same arguments suggest that that the dependence on the form $\omega$ is likewise $Q$-exact. This is more subtle, since $\omega$ can appear in the measure. We will return to this issue in section 7.2 below.

It is straightforward to introduce a coupling to a Beltrami differential coupling to $\int \bar{\mu} T$ with

$$
T=\langle b, \partial c\rangle+\langle\partial(\omega \bar{\phi}) \stackrel{\otimes}{\otimes} \partial \phi\rangle \in \Omega^{2,1}\left(T^{1,0 *} X\right)
$$

$T$ is essentially the stress-energy tensor with the volume form included. Since we have included the volume form we have index structure:

$$
T_{i j \bar{k} \ell}=b_{i j} \partial_{\ell} c_{\bar{k}}+\partial_{[i}\left(\omega_{j]} \bar{k} \bar{\phi}\right) \partial_{\ell} \phi
$$

Remarks:

1. In an entirely similar way, if $F^{2,0}(a)=0$, but $\bar{a}$ is arbitrary we can gauge fix the action

$$
\int \omega\left\langle\partial_{a} e, c\right\rangle
$$

The scalars become $(2,0),(0,2)$-forms and the current coupling to $\bar{a}$ is $Q$-exact.

2. Henceforth in this paper for simplicity we assume that $X$ is a Kähler manifold. This allows us to use the Kähler identities $\bar{\partial}^{\dagger}=[\Lambda, \partial]$ where $\Lambda$ is the contraction with the Kähler form. In section four we will integrate $\partial_{a}$ by parts and write formulae in terms of $(\bar{\partial})_{a}^{\dagger}$.

\subsection{Generalization to $2 n$ dimensions}

The generalization to $2 n$ dimensions is straightforward. It is easiest to work on a Kähler manifold. The gauge fixing procedure now produces a sequence of ghosts for ghosts. Schematically the Lagrangian has the form

$$
\int b\left(\bar{\partial}+\bar{\partial}^{\dagger}\right) c+\tilde{\phi} \bar{\partial} \bar{\partial}^{\dagger} \phi+\ldots
$$

Here $b \in \oplus \Omega^{2 p, 0}\left(E^{*}\right)$ while $c \in \Omega^{0,2 p-1}(E)$ (and for simplicity we have omitted powers of the Kahler form $\omega$ ). As we recall below the first term is simply the action for a Weyl fermion in a particular background gauge field. 


\section{3. $b c$ systems as supersymmetric field theories}

\subsection{Chiralmultiplets and vectormultiplets in $d=4$}

On a Kähler surface $X$ we can consider a chiral superfield $\Phi$ and twist it by a halfintegral twist with the $R$-current [11] [12] [13]. F The result is that we can turn the Weyl fermion into anticommuting form fields $\psi_{\alpha} \rightarrow c, \bar{\psi}^{\dot{\alpha}} \rightarrow b, e$. Put more mathematically, we consider a chiral superfield $\Phi$ in the bundle $E \otimes K^{-1 / 2}$. The conjugate superfield $\bar{\Phi}$ is in $E^{*} \otimes K^{+1 / 2}$, so, by a standard isomorphism, the fermions in the theory are sections:

$$
\begin{aligned}
& \psi_{\alpha} \in \Gamma\left[S^{-} \otimes K^{-1 / 2} \otimes E\right] \cong \Omega^{0,1}(E) \\
& \bar{\psi}^{\dot{\alpha}} \in \Gamma\left[S^{+} \otimes K^{1 / 2} \otimes E^{*}\right] \cong \Omega^{0,0}\left(E^{*}\right) \oplus \Omega^{2,0}\left(E^{*}\right)
\end{aligned}
$$

Thus from a free superfield we get exactly the field content of the $b c$ system. Moreover, under the isomorphism (3.1) the Dirac operator with spinor covariant derivative coupled to $K_{X}^{ \pm 1 / 2}$ :

$$
\begin{aligned}
& \nabla_{\mu}^{-}=\partial_{\mu}-\frac{1}{2} \omega_{\mu}^{a b}\left(\sigma^{a b}\right) \pm V_{\mu}+A_{\mu} \\
& \nabla_{\mu}^{+}=\partial_{\mu}-\frac{1}{2} \omega_{\mu}^{a b}\left(\bar{\sigma}^{a b}\right) \pm V_{\mu}+A_{\mu}
\end{aligned}
$$

where $V_{\mu}=\partial_{\mu} \log \sqrt{g}$ is mapped to the Dolbeault operator according to $\bar{\sigma} \cdot \nabla^{-} \rightarrow \bar{\partial}_{\bar{a}} \oplus \bar{\partial}_{\bar{a}}^{\dagger}$ for $K_{X}^{-1 / 2}$, etc. Thus coupling to $K^{-1 / 2}$ is equivalent to topological twisting by the $R$-current. The action:

$$
\int \sqrt{g}\left[g^{\mu \nu}\left\langle\nabla_{\mu} \bar{\phi}, \nabla_{\nu} \phi\right\rangle+i\left\langle\bar{\psi}_{\dot{\alpha}}, \bar{\sigma}^{\mu \dot{\alpha} \alpha} \nabla_{\mu}^{-} \psi_{\alpha}\right\rangle\right]
$$

is equivalent to (2.7) if $f \equiv g^{i \bar{j}} F_{i \bar{j}}=0$. More generally, if we consider the coupled vectormultiplet-chiralmultiplet system in a vectormultiplet background with $\lambda=F^{0,2}=0$ (where $\lambda$ is the gluino) the standard Lagrangian and the $b c$ Lagrangian differ by $Q$-exact terms. Finally the supercurrent $\bar{q}^{\dot{\alpha}, \mu}=\left\langle\bar{\sigma}^{\nu} \sigma^{\mu} \bar{\psi}, \nabla_{\nu} \phi\right\rangle$ is conserved when the background SYM fields are antiself-dual. Using the projectively covariantly constant spinor on a Kahler manifold we can produce the operator $Q$ from $\bar{Q}^{\dot{A}}$ :

$$
Q=\int_{X_{3}} \bar{s}_{\dot{\alpha}} \bar{q}^{\dot{\alpha}} n_{\mu}
$$

Even if $f \neq 0 Q$ is still a conserved charge as long as $F^{0,2}=0$. We now recognize the $b c$ system as a twisted Euclidean chiral supermultiplet in a supersymmetry-preserving background.

One may also consider the $b c$ system corresponding to (2.16). Its partition function turns out to be related to that of a vectormultiplet, expanded in background field gauge.

5 We work in Euclidean space so fermions are Weyl fermions. 


\subsection{Hypermultiplets and vectormultiplets in $d=6$}

The Lagrangians for hypermultiplets and vectormultiplets on Kähler manifolds in six dimensions can be related to $b c$ systems along the above lines.

In $d=6, \mathcal{N}=(1,0)$ supersymmetry a hypermultiplet consists of Bose/Fermi fields $\Phi$ and $\psi$ transforming in in the $S U(4) \times U S p(2)_{\mathcal{R}}$ Lorentz $\times$ R-symmetry group as $(1 ; 2)$ and $(4 ; 1)$ respectively. On a Kahler manifold the holonomy group is $U(3)$. Twisting by a linear combination of the $U(1)$ in $U(3)$ and the internal symmetries we produce the Lagrangian

$$
\begin{gathered}
I=\int_{X_{6}} \omega b^{2,0} \bar{\partial} c^{0,1}+\omega\left\langle b^{2,0}, \bar{\partial}^{\dagger} c^{0,3}\right\rangle+\omega^{3}\left\langle b^{0,0}, \bar{\partial}^{\dagger} c^{0,1}\right\rangle \\
+\int_{X_{6}} \omega^{3}\left\langle\tilde{\phi}^{0,0}, \bar{\partial}^{\dagger} \bar{\partial} \phi^{0,0}\right\rangle+\left\langle\partial \partial^{\dagger} \bar{\phi}^{3,0}, \phi^{0,3}\right\rangle
\end{gathered}
$$

where superscripts indicate the form type.

By choosing an appropriate twisting we have a scalar $Q$-symmetry:

$$
\begin{aligned}
Q: c^{0,1} & \rightarrow \bar{\partial} \phi^{0,0} & \phi^{0,0} & \rightarrow 0 \\
\bar{\phi}^{0,0} & \rightarrow-b^{0,0} & b^{0,0} & \rightarrow 0 \\
b^{2,0} & \rightarrow \partial^{\dagger} \bar{\phi}^{3,0} & \bar{\phi}^{3,0} & \rightarrow 0 \\
\phi^{0,3} & \rightarrow c^{0,3} & c^{0,3} & \rightarrow 0
\end{aligned}
$$

such that the action is:

$$
I=\int \omega b^{2,0} \bar{\partial} c^{0,1}+Q\left(\int \omega^{2} \partial \bar{\phi}^{0,0} c^{0,1}+\partial b^{2,0} \phi^{0,3}\right)
$$

In much the same way, by gauge fixing

$$
\int b^{3,0} \bar{\partial} c^{0,2}
$$

we produce the Lagrangian used for evaluating a $d=6, \mathcal{N}=(1,0)$ vectormultiplet in background field gauge.

\section{3. $d=2 n$}

On a Kahler manifold there is a standard relation between the Dirac operator coupled to a vector bundle $E \otimes K_{X}^{ \pm 1 / 2}$ and the Dolbeault operator coupled to $E$ [20]. Given an Hermitian vector bundle on an arbitrary Kahler manifold:

$$
\begin{aligned}
\Gamma\left[S^{+} \otimes K^{1 / 2} \otimes E\right] & \cong \oplus \Omega^{2 p, 0}(E) \\
\Gamma\left[S^{-} \otimes K^{1 / 2} \otimes E\right] & \cong \oplus \Omega^{2 p-1,0}(E) \\
\Gamma\left[S^{+} \otimes K^{-1 / 2} \otimes E\right] & \cong \oplus \Omega^{0,2 p}(E) \\
\Gamma\left[S^{-} \otimes K^{-1 / 2} \otimes E\right] & \cong \oplus \Omega^{0,2 p-1}(E)
\end{aligned}
$$


Under this isomorphism the Weyl operator becomes the Dolbeault operator according to

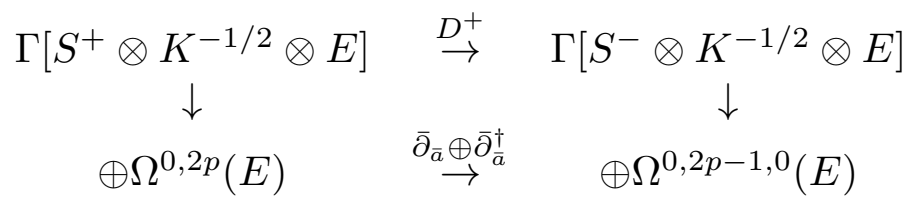

Accordingly, the physical content of all higher-dimensional $b c$ systems consists of a twisted Weyl fermion together with a set of $(0, p)$-form bosons. When coupled to a nontrivial vector bundle these theories are (noninteracting!) nonabelian $p$-form theories.

\section{Defining the chiral partition function}

\subsection{Perturbation theory point of view}

The most straightforward definition of the path integral of the $b c$ system is as the generating functional for current correlation functions:

$$
Z_{c h, s}[a, \bar{a}, \mu, \bar{\mu}]=\left\langle\exp \int \operatorname{Tr}(\bar{a} J)+\bar{\mu} T\right\rangle
$$

defined by perturbation theory in $\bar{a}, \bar{\mu}$. Since the theory is simply a theory of a Weyl fermion together with a collection of bosons we can use the standard perturbation series of quantum field theory. The propagators can be defined, for example, by introducing a Dirac fermion with left and right currents coupled to different gauge fields. In the present case we expand in $\bar{a}, \bar{\mu}$ and use the free propagators:

$$
\begin{aligned}
& \left\langle\phi^{m}(x) \omega(y) \partial_{y} \bar{\phi}_{n}(y)\right\rangle=\frac{i \delta_{n}^{m}{ }_{n}}{4 \pi^{2}} \frac{(\bar{x}-\bar{y})^{\bar{i}} \epsilon_{\bar{i} \bar{j}} d \bar{y}^{\bar{j}}}{(x-y)^{4}} d y^{1} d y^{2} \\
& \left\langle c^{m}(x) b_{n}(y)\right\rangle=\frac{\delta^{m}{ }_{n}}{\pi^{2}} \frac{(\bar{x}-\bar{y})^{\bar{i}} \epsilon_{\bar{i} \bar{j}} d \bar{x}^{\bar{j}}}{(x-y)^{4}} d y^{1} d y^{2}
\end{aligned}
$$

This procedure plainly produces a holomorphic function of $\bar{a}, \bar{\mu}$. Of course, this procedure also produces infinities from divergences at coincident points. Thus the chiral perturbation series in fact depends on extra data, as is standard in quantum field theory. For example, defining the short-distance expansion of a Green's function requires comparing two fibers of a vector bundle at nearby, but different points. It is thus natural to introduce a complete connection $A=(a, \bar{a})$ when subtracting infinities.

Because of supersymmetric cancellations the leading term in the expansion is cubic in $A$. The resulting functional is of course anomalous under gauge transformations because of the Weyl fermion. The bosons only contribute to the anomaly through a coboundary. In what follows we assume that the theory can be quantized with the standard formula for the gauge anomaly of Weyl fermions [14] [15] [16]. 


\subsection{Factoring Determinants point of view}

The partition function (4.1) is formally evaluated by writing out the free field modes.:

$$
\mathcal{Z}_{c h} \stackrel{?}{=} \int\left[\operatorname{dedbdc]}[d \phi d \tilde{\phi}] \exp \left\{-S_{b c}\right\}=\frac{\operatorname{Det}^{\prime}\left[\left(\bar{\partial}_{\bar{a}} \oplus(\bar{\partial})_{a}^{\dagger}\right)\right]_{(0,1)}}{\operatorname{Det}^{\prime}\left[(\bar{\partial})_{a}^{\dagger} \bar{\partial}_{\bar{a}}\right]_{0,0}}\right.
$$

in four dimensions, with a simple generalization to $2 n$-dimensions. In this paper we ignore moduli so zeromode insertions are not needed. As we have emphasized throughout the paper, if there is no $Y$-anomaly then in fact $\mathcal{Z}_{c h}$ is only a function of $\bar{a}$.

The expression (4.3) is formal. Any definition using determinants must face up to the fact that $\bar{\partial}+\bar{\partial}^{\dagger}$ is an operator between different spaces. The time-honored approach to this difficulty is the introduction of a system of the opposite chirality: $\bar{b} \in \Omega^{0,2}(E), \bar{c} \in$ $\Omega^{1,0}\left(E^{*}\right)$ etc. In terms of the Weyl fermions we are introducing the complex conjugate

representation. We can define a measure on the resulting theory. 6 Coupling the opposite chirality to the gauge fields $(b, \bar{b})$ we obtain the general nonchiral system:

$$
\mathbb{Z}[a, \bar{a} ; b, \bar{b}] \equiv \frac{\operatorname{Det}\left[\left(\bar{\partial}_{\bar{b}}+\left(\bar{\partial}^{\dagger}\right)_{b}\right)\left(\bar{\partial}_{\bar{a}}+\left(\bar{\partial}^{\dagger}\right)_{a}\right)\right]_{0,1}}{\operatorname{Det}\left[\left(\bar{\partial}^{\dagger}\right)_{a} \bar{\partial}_{\bar{a}}\right]_{0,0} \operatorname{Det}\left[\left(\bar{\partial}^{\dagger}\right)_{b} \bar{\partial}_{\bar{b}}\right]_{0,0}}
$$

From the Q-symmetry of the classical action for nonchiral system (provided that $\left.F^{(0,2)}(\bar{a})=F^{(2,0)}(b)=0\right)$ we conclude that

$$
\log \mathbb{Z}[a, \bar{a} ; b, \bar{b}]=\log \mathcal{Z}_{\text {nonchiral }}[\bar{a}, b]+\mathcal{C}_{\text {local }}[a, \bar{a} ; b, \bar{b}]
$$

where $\mathcal{C}_{\text {local }}[a, \bar{a} ; b, \bar{b}]$ is a local counterterm. Note that we do not impose any conditions on gauge fixing fields $a, \bar{b}$.

In order to compute nonchiral function $\mathcal{Z}_{\text {nonchiral }}[\bar{a}, b]$ we will take $a=b$, and $\bar{b}=\bar{a}$, so that we get, up to local counterterms:

$$
\mathcal{Z}_{\text {nonchiral }}[\bar{a}, b]=\frac{\operatorname{Det}\left[\left\{\left(\bar{\partial}^{\dagger}\right)_{b}, \bar{\partial}_{\bar{a}}\right\}\right]_{0,1}}{\left(\operatorname{Det}\left[\left(\bar{\partial}^{\dagger}\right)_{b} \bar{\partial}_{\bar{a}}\right]_{0,0}\right)^{2}}
$$

The right hand side of this equation is nothing but Ray-Singer (RS)-torsion, and the problem of computation of chiral part of the partition functon is therefore just the problem of factorization of RS-torsion into a product of terms depending only on $\bar{a}$ and $b$ up to local counterterm:

$$
\mathcal{Z}_{\text {nonchiral }}[\bar{a}, b]=\mathcal{Z}_{c h}(\bar{a}) \overline{\mathcal{Z}}_{c h}(b) \exp \mathcal{C}_{\text {local }}[\bar{a}, b]
$$

We will study the generalized RS-torsions (containing $\mu$ ) their factorizations and corresponding gauge and diffeomorphism anomalies of chiral systems with the help of supersymmetric quantum mechnanics in the next section.

6 For a real representation on a hyperkahler manifold we could define a mass term using the nowhere-vanishing $(2,0)$ form. 


\subsubsection{Determinant lines and Quillen norms}

There is a well-developed mathematical theory of the objects $(4.3)$ : this is the theory of determinant lines and Quillen metrics. $\mathcal{Z}_{c h}$ should be regarded as a section of a determinant line bundle $\lambda$ over a parameter space $\mathcal{S}$. In this case $\lambda$ is dual to the line bundle

$$
\Lambda^{m x} H_{\bar{\partial}}^{0,0}(X ; E) \otimes\left(\Lambda^{m x} H_{\bar{\partial}}^{0,1}(X ; E)\right)^{-1} \otimes \Lambda^{m x} H_{\bar{\partial}}^{0,2}(X ; E)
$$

where $\Lambda^{m x}$ is the maximum exterior power. While $\lambda$ is canonically trivial (absence of zeromodes) it carries a highly nontrivial Quillen metric:

$$
\left\|\mathcal{Z}_{c h}\right\|^{2} \equiv \frac{\operatorname{Det}^{\prime}\left[\left(\bar{\partial}_{\bar{a}}\right)^{\dagger} \bar{\partial}_{\bar{a}}\right]_{0,1}}{\operatorname{Det}^{\prime}\left[\left(\bar{\partial}_{\bar{a}}\right)^{\dagger} \bar{\partial}_{\bar{a}}\right]_{0,0}}
$$

which is again RS-torsion [21]. The zeromodes are easily reinstated in the above formulae. The above remarks generalize straightforwardly to the $2 n$-dimensional case.

It turns out, as we will demonstrate in the next section that the nonchiral partition function can be split holomorphically:

$$
\mathcal{Z}_{\text {nonchiral }}[\bar{a}, a]=\left\|\mathcal{Z}_{c h}\right\|^{2}=e^{\Gamma[a, \mu]+K(a, \bar{a} ; \mu, \bar{\mu})+\Gamma(\bar{a}, \bar{\mu})}
$$

where $\Gamma$ is a nonlocal, but holomorphic functional, while $K$ is a local, but nonholomorphic functional. $\exp \Gamma$ defines the chiral partition function $\mathcal{Z}_{c h}$.

\section{Three derivations of the chiral partition function}

In this section we describe three methods to evaluate the partition function for $\bar{a}, \bar{\mu}$ satisfying (1.3).

\subsection{Anomaly plus holomorphy}

For simplicity we work in four dimensions, put $\bar{\mu}=0$, and consider the definition of the chiral partition function via the perturbation expansion. The process of gauge fixing introduces a $(1,0)$ connection $a$. Let us assume that the perturbation expansion (4.1) can be regularized so that under unitary gauge transformations we have the standard anomalous variation:

$$
Z_{c h, s}\left(a^{g}, \bar{a}^{g}\right)=e^{i \alpha_{s}(a, \bar{a}, g)} Z_{c h, s}(a, \bar{a})
$$


for a cocycle $\alpha_{s}$ derived from the descent procedure. Explicitly, in 4-dimensions we have the standard formula

$$
\begin{aligned}
& \alpha_{s}(\bar{a}, a, g)=\frac{1}{240 \pi^{2}} \int_{X_{4} \times I} \operatorname{Tr}\left(g^{-1} d g\right)^{5} \\
& \quad-\frac{1}{48 \pi^{2}} \int_{X_{4}} \operatorname{Tr}\left[\left(A d A+d A A+A^{3}\right) d g g^{-1}-\left(A d g g^{-1}\right)^{2}-A\left(d g g^{-1}\right)^{3}\right]
\end{aligned}
$$

where $A=a+\bar{a}$ and with the corresponding Lie algebra cocycle ( for $g=1+\epsilon+\cdots$ ) given by: $\int \operatorname{Tr} \epsilon\left[2(d A)^{2}+d\left(A^{3}\right)\right]$. Evidently, the standard cocycle depends on both $a$ and $\bar{a}$, so the standard regularization violates BRST decoupling. Nevertheless, one can introduce the counterterm:

$$
\gamma(a, \bar{a})=\int \operatorname{Tr}\left[(2 \partial \bar{a}+\bar{\partial} a)(a \bar{a}-\bar{a} a)+\frac{3}{2}(a \bar{a})^{2}\right]
$$

and check, by direct calculation, that

$$
\int \operatorname{Tr} \epsilon\left[2(d A)^{2}+d\left(A^{3}\right)\right]-\delta_{\epsilon} \gamma(a, \bar{a})=6 \int \operatorname{Tr} \epsilon(\partial \bar{a})^{2}
$$

Therefore the chiral partition function $\mathcal{Z}_{c h}(\bar{a}) \equiv e^{i \gamma(a, \bar{a})} \mathcal{Z}_{c h, s}(a, \bar{a})$ satisfies:

$$
\mathcal{Z}_{c h}\left(\bar{a}^{g}\right)=e^{i \alpha_{h}(\bar{a}, g)} \mathcal{Z}_{c h}(\bar{a})
$$

with the group cocycle:

$$
\alpha_{h}(\bar{a}, g)=\alpha_{s}(a, \bar{a}, g)+\delta \gamma=\alpha_{s}(a, \bar{a}, g)+\left(\gamma\left(a^{g}, \bar{a}^{g}\right)-\gamma(a, \bar{a})\right)
$$

Now we can analytically continue formula (5.5) from unitary to complex transformations and (locally) gauge away $\bar{a}: \bar{a}=g^{-1} \bar{\partial} g$. Thus the $\bar{a}$-dependence of the chiral partition function is given exactly by the effective action:

$$
\mathcal{Z}_{c h}(\bar{a})=e^{\Gamma[\bar{a}]}=e^{i \alpha_{h}(0, g)}
$$

This is a central result of this paper. Later we will give two more derivations of this formula and will also include gravity (via the diffeomorphism dependence of the chiral partition function).

Recall that in two-dimensions the trivial cocycle is also fixed by holomorphy and leads to the kinetic term in the $W Z W_{2}$ action. In $4 \mathrm{~d}$ there is an analogous term but it is of dimension four. An ambiguous term $\int \operatorname{Tr} \bar{a} \alpha$ remains, where $\alpha$ is a 2, 1 form. (For example, it could be that $\alpha \sim \omega \partial K_{0}$ for the background Kahler metric.)

Remark: On general grounds one expects that in $2 n$ dimensions there is a local counterterm $\gamma(a, \bar{a})$ satisfying (5.6). This counterterm should be closely related to the "Bardeen counterterm" relating VA and LR forms of the anomaly [16]. In any case, the explicit form of $\gamma(a, \bar{a})$ is not needed for the construction of the chiral cocycle Lagrangians below. 


\subsection{Family index theorem and the Quillen anomaly}

Let us now recall the topological interpretation of anomalies following from the family index theorem [22] 20] 23]. The (differential form version) of the family index theorem has

already been applied in this context in [24] 25] and, most definitively, by Bismut, Gillé and Soulet [21]. The determinant line bundle $\lambda \rightarrow \mathcal{S}$ over a parameter space $\mathcal{S}$ has first Chern class:

$$
\partial^{(\mathcal{S})} \bar{\partial}^{(\mathcal{S})} \log \left\|\mathcal{Z}_{c h}\right\|^{2}=(2 \pi i)\left[\int_{X} \operatorname{Td}\left(T^{1,0} Z\right) \operatorname{ch}(\hat{E})\right]^{(2)}
$$

where $Z \rightarrow \mathcal{S}$ is fibered by $X, T^{1,0} Z$ is the relative tangent bundle, and $\hat{E}$ is the universal bundle. In fact, using $\zeta$-function regularization we may choose the Bismut-Freed connection on $\lambda$ and obtain an explicit differential form representative of the RHS of (5.8). When the parameter space is a quotient by a gauge group e.g. $\mathcal{S}=\mathcal{A} / \mathcal{G}$ then the line bundle $\lambda$ descends from a $\mathcal{G}$-equivariant line bundle on $\mathcal{A}$. The curvature is directly related to the equivariance of $Z(a, \bar{a})$ under gauge transformations.

One may explicitly evaluate the RHS of (5.8) using the techniques explained in the next section. Putting $R=0$ for simplicity we find, by direct computation, that (5.8) has the form $\partial^{(\mathcal{S})} \bar{\partial}^{(\mathcal{S})} \beta(a, \bar{a})$ where

$$
\begin{array}{rlrl}
\beta(a, \bar{a}) & =\frac{1}{2 \pi} \int_{X_{2}} \operatorname{Tr} a \bar{a} \quad n=1 \\
& =\frac{1}{8 \pi^{2}} \int_{X_{4}} \operatorname{Tr}\left(\bar{a} a \bar{\partial} a-a \bar{a} \partial \bar{a}+\frac{1}{2}(\bar{a} a)^{2}\right) & n=2
\end{array}
$$

Thus we have justified (4.10) with $K(a, \bar{a})=\beta(a, \bar{a})$. Of course, the full partition function is (unitarily) gauge invariant while $\beta$ is not. Indeed from the the gauge transformations of $\beta$ one can derive the holomorphic action $\Gamma[\bar{a}]$ :

$$
\delta \beta(a, \bar{a}, g)=-\delta \bar{\Gamma}[a, g]-\delta \Gamma[\bar{a}, g]
$$

and hence the derive chiral partition function. We will describe the formula in the next section. 


\subsection{Derivation from supersymmetric quantum mechanics}

One rather direct way to prove the index theorems uses supersymmetric quantum mechanics [26] [27]. Using the techniques used to prove the index theorems one can also study the Lie algebra cocycles. This method is very simple and, in the case of diffeomorphism cocycles is the most direct route to the answer.

As is well-known Ray-Singer torsion can be defined using a trace in a supersymmetric quantum mechanics system with bosonic coordinates $\phi^{i}, \bar{\phi}^{\bar{i}}$ and corresponding fermionic coordinates $\psi^{i}, \bar{\psi}^{\bar{i}}$. Suppose we have two $\bar{\partial}$-operators: $\left(\bar{\partial}+\bar{\mu}_{1} \cdot \partial+\bar{a}_{1}\right)^{2}=0$, and $\left(\bar{\partial}+\bar{\mu}_{2}\right.$. $\left.\partial+\bar{a}_{2}\right)^{2}=0$. Introducing a reference Kahler metric $g^{(0)}$ we can take $Q_{1}=\bar{\partial}+\bar{\mu}_{1} \cdot \partial+\bar{a}_{1}$ and $\bar{Q}_{2}=\left(\bar{\partial}+\bar{\mu}_{2} \cdot \partial+\bar{a}_{2}\right)^{\dagger}$ as right and left arrows respectively in the complexes:

$$
\begin{aligned}
& 0 \longrightarrow \Omega^{0,0} \stackrel{Q_{1}}{\longrightarrow} \Omega^{0,1} \stackrel{Q_{1}}{\longrightarrow} \Omega^{0,2} \stackrel{Q_{1}}{\longrightarrow} \cdots \Omega^{0, n} \longrightarrow 0 \\
& 0 \longleftarrow \Omega^{0,0} \stackrel{\bar{Q}_{2}}{\longleftarrow} \Omega^{0,1} \stackrel{\bar{Q}_{2}}{\longleftarrow} \Omega^{0,2} \stackrel{\bar{Q}_{2}}{\longleftarrow} \cdots \Omega^{0, n} \longleftarrow 0
\end{aligned}
$$

This complex can be realized in the context of supersymmetric quantum mechanics: $\oplus \Omega^{0, *}$ is the Hilbert space of states, $Q_{1}, \bar{Q}_{2}$ are supersymmetry operators, there is a fermion number operator $F$ such that $\left\{F, Q_{1}\right\}=Q_{1},\left\{F, \bar{Q}_{2}\right\}=-Q_{2}$, and $H=\left\{Q_{1}, \bar{Q}_{2}\right\}$. The regularized nonchiral partition function, generalizing (4.9), can be defined as:

$$
\log \mathcal{Z}_{\text {nonchiral }}=\int_{\Lambda^{-1}}^{\infty} \frac{d t}{t} \operatorname{Tr}(-1)^{F} F e^{-t H}
$$

Subtracting the divergences for $\Lambda^{-1} \rightarrow 0$ introduces the standard ambiguity by local counterterms.

Using standard manipulations and the properties $Q_{1}^{2}=\bar{Q}_{2}^{2}=0$ we find that under a variation $\bar{Q}_{2} \rightarrow \bar{Q}_{2}+\left[\bar{Q}_{2}, \epsilon\right]$ holding $Q_{1}$ fixed, we have:

$$
\delta_{\epsilon}^{0,1} \log \mathcal{Z}_{\text {nonchiral }}=\delta_{\epsilon}^{0,1} \int_{\Lambda^{-1}}^{\infty} \frac{d t}{t} \operatorname{Tr}(-1)^{F} F e^{-t H}=\lim _{\Lambda \rightarrow \infty} \operatorname{Tr}(-1)^{F} \epsilon e^{-H / \Lambda}
$$

Let us give two examples of results easily obtained in this way.

\subsubsection{Gauge cocycle}

First, let us put $\bar{\mu}=0$, work in $2 n$ dimensions with a Euclidean metric, and consider "chiral gauge transformations" $(a, \bar{a}) \rightarrow\left(a, \bar{a}+\bar{\partial}_{\bar{a}} \epsilon\right)$. We then recognize (5.13) as the 
formula used in the evaluation of anomalies using the "Fujikawa method," and hence the answer for the infinitesimal cocycle follows immediately: $\mathrm{O}$

$$
\frac{1}{(2 \pi)^{n} n !} \int_{X_{2 n}} \operatorname{Tr} \epsilon(F(a, \bar{a}))^{n}
$$

As in the previous two sections we observe a violation of $Q$-symmetry since the infinitesimal cocycle depends on $a$, and as in the previous two sections we can add a local counterterm to remedy this. 6 The resulting cocycle is simply obtained by putting $a=0$ to give:

$$
C(\epsilon, \bar{a})=\left.\frac{d}{d \xi}\right|_{\xi=0}\left[(2 \pi i) \int_{X} \operatorname{ch}\left(d \bar{z}^{\bar{a}} d z^{c} F_{\bar{a} c}+\xi \epsilon\right)\right]=\frac{1}{(2 \pi i)^{n} n !} \int_{X_{2 n}} \operatorname{Tr} \epsilon(\partial \bar{a})^{n}
$$

\subsubsection{Diffeomorphism cocycle}

We now indicate how one may include the $\bar{\mu}$ dependence in our results. For simplicity we take the simplest case of $\bar{a}_{1}=\bar{a}_{2}=\bar{\mu}_{2}=0$, but work in $2 n$ dimensions. In our supersymmetric quantum mechanics the bosonic coordinates are $\phi^{i}, \bar{\phi}^{\bar{i}}$ with fermionic partners $\psi^{i}, \bar{\psi}^{\bar{i}}$ and Q-symmetries:

$$
\begin{aligned}
Q_{1}: \bar{\phi}^{\bar{i}} \rightarrow \bar{\psi}^{\bar{i}} & \bar{\psi}^{\bar{i}} \rightarrow 0 \\
\phi^{i} \rightarrow \bar{\psi}^{\bar{j}} \bar{\mu}_{\bar{j}}^{i} & \psi^{i} \rightarrow \dot{\phi}^{i}-\dot{\bar{\phi}}^{\bar{j}} \bar{\mu}_{\bar{j}}^{i}-\psi^{k} \bar{\psi}^{j} \partial_{k} \bar{\mu}_{\bar{j}}^{i} \\
\bar{Q}_{2}: \phi^{i} \rightarrow \psi^{i} & \bar{\phi}^{\bar{i}} \rightarrow 0 \\
\psi^{i} \rightarrow 0 & \bar{\psi}^{\bar{i}} \rightarrow \dot{\bar{\phi}}^{\bar{i}}
\end{aligned}
$$

One can easily check that $Q_{1}^{2}=\bar{Q}_{2}^{2}=0$ using the Kodaira-Spencer equation. Moreover, $\left\{Q_{1}, \bar{Q}_{2}\right\}$ acts on the fields as $\frac{d}{d t}$ and the action is

$$
S_{S Q M}=\left\{\bar{Q}_{2},\left\{Q_{1}, \int \dot{\phi}^{i} \partial_{i} K\right\}\right\}
$$

where $K$ is the Kahler potential.

The result for (5.13) is easily obtained following closely the manipulations in section 11 of [28]. The result is simply expressed in terms of the Todd class with the curvature (in holomorphic frame indices $a, b, \ldots$ ) shifted by:

$$
R_{b \bar{a} c}^{d} \rightarrow R_{b \bar{a} c}^{d}+\partial_{b} \partial_{c} \bar{\mu}_{\bar{a}}^{d}
$$

7 Note that this appears to be a consistent, not a covariant anomaly. However, under holomorphic gauge transformations acting only on $\bar{a}$ it is in fact a consistent anomaly.

8 The explicit form of this counterterm, $\beta(a, \bar{a})$ is given in the next section. 
Explicitly, taking the metric to be flat:

$$
C(v, \bar{\mu})=\left.\frac{d}{d \xi}\right|_{\xi=0}\left[\int_{X} T d\left(d \bar{z}^{\bar{a}} d z^{c} \partial_{b} \partial_{c} \bar{\mu}_{\bar{a}}{ }^{d}+\xi \partial_{b} v^{d}\right)\right]
$$

\subsubsection{Integrated anomaly}

Combining (5.13) and (5.14) we obtain the nonchiral partition function $\mathcal{Z}_{\text {nonchiral }}[\bar{a}, a]$ by integrating both variations. Putting $a=0$ we can then obtain the chiral partition function. The resulting action is described in section seven below. Similar remarks apply to the diffeomorphism case.

\section{Holomorphic descent and Bott-Chern classes}

In the previous section we have argued that the chiral $b c$ partition function is simply expressed in terms of a group cocycle for the complexified gauge group taking values in functionals of differential operators $\bar{\partial}_{\bar{a}}$ squaring to zero. The proper mathematical objects for understanding this are Bott-Chern holomorphic secondary characteristic classes [29] 24] 25] [21].

Let us begin by recalling the usual construction of secondary characteristic classes. If $P(F)$ is an invariant polynomial on the Lie algebra $\underline{g}$ of a gauge group then, under an arbitrary variation of the gauge field:

$$
\delta P(F)=d\left[P^{\prime}(\delta A, F)\right]
$$

Integrating this along a path between two connections gives the Chern-Simons form:

$$
d C S\left[A_{1} ; A_{0}\right]=P\left(F_{1}\right)-P\left(F_{0}\right)
$$

From gauge variation of $A_{1}$, holding $A_{0}$ fixed we obtain the standard descent tower:

$$
\begin{gathered}
\delta_{\epsilon} C S\left[A_{1} ; A_{0}\right]=d \omega_{2 n}^{1}\left[\epsilon, A_{1}, F_{1}\right] \\
\ldots \ldots
\end{gathered}
$$

leading to the standard group cocycle for gauge anomalies. 


\subsection{Bott-Chern classes}

Let us now assume that $X$ has a complex structure and that $F$ is of type $(1,1)$ : that is $(a, \bar{a}) \in \mathcal{A}^{1,1}$, may be written as:

$$
\begin{aligned}
& a=g_{L}^{-1} \partial g_{L} \\
& \bar{a}=-\left(\bar{\partial} g_{R}\right) g_{R}^{-1}
\end{aligned}
$$

For a unitary connection $g_{R}=g_{L}^{\dagger}$, but we will not assume this in general. This connection defines a holomorphic vector bundle $\mathcal{E}$ with holomorphic framing defined by $\bar{\partial}_{\bar{a}} \overrightarrow{\mathbf{e}}=0$. It follows from (6.4) that $F=g_{R} \bar{\partial}\left(h^{-1} \partial h\right) g_{R}^{-1}=-g_{L}^{-1} \partial\left(\bar{\partial} h h^{-1}\right) g_{L}$ where $h=g_{L} g_{R}$. For unitary connections $h$ is a hermitian metric on the holomorphic bundle $\mathcal{E}$. Thus we may equivalently think of the background data as a connection in $\mathcal{A}^{1,1}$ or as a quadratic form on a holomorphic bundle $\mathcal{E}$. Unitary connections correspond to positive hermitian forms.

Using the complex structure we may decompose

$$
\delta P(F)=\bar{\partial}\left[P^{\prime}\left(\delta a^{1,0}, F\right)\right]+\partial\left[P^{\prime}\left(\delta \bar{a}^{0,1}, F\right)\right]
$$

under an arbitrary variation of connection. Moreover, if we are taking a variation of $A$ within $\mathcal{A}^{1,1}$ then the variation is formally equivalent to a gauge transformation:

$$
\begin{aligned}
\delta_{\epsilon_{L}}^{0,1} a=\partial_{a} \epsilon_{L} & \delta_{\epsilon_{L}}^{0,1} \bar{a}=0 \\
\delta_{\epsilon_{R}}^{1,0} a=0 & \delta_{\epsilon_{R}}^{1,0} \bar{a}=-\bar{\partial}_{\bar{a}} \epsilon_{R}
\end{aligned}
$$

and hence, by the descent formalism a variation $\delta P(F)$ along $\mathcal{A}^{1,1}$ is in fact $\partial \bar{\partial}$ exact:

$$
\delta P(F)=\left(\delta^{1,0}+\delta^{0,1}\right) P(F)=\bar{\partial} \partial \delta \mathcal{R}(h)
$$

Integrating this equation along a path gives the Bott-Chern holomorphic secondary classes:

$$
\bar{\partial} \partial \mathcal{R}\left(h_{1} ; h_{0}\right)=P\left(F\left(h_{1}\right)\right)-P\left(F\left(h_{0}\right)\right)
$$

which should be viewed as the analog of (6.2). The functional $\exp \int_{X_{2 n}} \mathcal{R}\left(h_{1} ; h_{0}\right)$ is the ratio of two nonchiral partition functions of the type described in the previous section. 


\subsection{Holomorphic descent}

It is important to stress that the Bott-Chern classes are not precisely the group cocycles that we want. These lead to the infinitesimal gauge cocycles

$$
\int \operatorname{Tr} \epsilon F(a, \bar{a})^{n}
$$

Recall that we must maintain $Q$-symmetry, and consequently $a$-independence. This is easily remedied by adding the coboundary $\beta$ mentioned in the previous section. Indeed, let $(a, \bar{a})$ be on $\mathcal{A}^{1,1}$. Then

$$
\operatorname{Tr}\left(F^{n}\right)=\operatorname{Tr}(\partial \bar{a})^{n}+\operatorname{Tr}(\bar{\partial} a)^{n}+\bar{\partial} \partial \beta(a, \bar{a})
$$

where $\beta$ is local and given explicitly by:

$$
\begin{gathered}
\beta(a, \bar{a})=\int_{0}^{1} d t \int_{0}^{1} d s \sum_{j=0}^{[(n-2) / 2]} \\
\frac{\left(t^{2}-t\right)^{j}\left(s^{2}-s\right)^{j}}{(j !)^{2}} \operatorname{Str}\left[\left(a^{2}\right)^{j},\left(\bar{a}^{2}\right)^{j}, a, \bar{a},\left(F_{s, t}\right)^{n-2 j-2}\right] \\
F_{s, t}=t \bar{\partial} a+s \partial \bar{a}+s t[a, \bar{a}]
\end{gathered}
$$

where $S t r$ is the graded symmetrized trace. This can be more elegantly written as:

$$
\int_{0}^{\infty} d \tau e^{-\tau} \int_{X_{2 n} \times I \times I} \operatorname{Tr} \exp \left[\mathcal{F}^{0,2}+\mathcal{F}^{2,0}+\tau \mathcal{F}^{1,1}\right]
$$

where $A(x, s, t)=t a(x)+s \bar{a}(x)$ and $d s, d t$ have type $(1,0)$ and $(0,1)$ respectively.

The addition of $\delta^{1,0} \beta$ (where $\delta^{1,0}$ takes a gauge variation of $\bar{a}$ holding $a$ fixed) converts (6.9) to the holomorphic cocycle

$$
\int \operatorname{Tr} \epsilon(\partial \bar{a})^{n}
$$

The condition $F^{0,2}(\bar{a})=0$ is crucial here.

The infinitesimal cocycle (6.13) can also be derived directly by the following holomorphic version of the descent procedure. Again let $P$ be an invariant polynomial on the Lie algebra $\underline{\text { g. }}$. We consider the $(n+1, n+1)$-form $P(\partial \bar{a})$. We assume $F^{0,2}=0$ so it is $\partial$ and $\bar{\partial}$ closed. Under an infinitesimal gauge transformation

$$
P(\partial \bar{a}) \rightarrow \bar{\partial} \partial \wp(\epsilon, \partial \bar{a})
$$

The $(n, n)$ form $\wp(\epsilon, \partial \bar{a})$ is a Lie algebra cocycle. Equivalently, we may write:

$$
P(\partial \bar{a})=\partial P_{1}(\bar{a}, \partial \bar{a})
$$

and, under gauge transformation $\bar{a} \rightarrow \bar{a}+\bar{\partial}_{\bar{a}} \epsilon$ :

$$
P_{1} \rightarrow P_{1}+\bar{\partial}[\wp(\epsilon, \partial \bar{a})]+\partial \xi^{n-1, n+1}
$$

where $\xi^{n-1, n+1}$ is some form. In this way we recover the holomorphic cocycle $\wp$. 


\section{Chiral Cocycle Theories}

In this section we briefly examine the effective actions induced by $b c$ systems. These generalize the $W Z W$ theories of two and four dimensions.

\subsection{Gauge transformations}

From the infinitesimal cocycle of the previous section we obtain the equation of motion 9

$$
\left(\bar{\partial}\left(g^{-1} \partial g\right)\right)^{n}=0
$$

The corresponding group cocycle is easily written as follows. Let us extend the field $g$ from $X_{2 n}$ to $X_{2 n} \times D$ where $D$ is a disk and $g=1$ on the outer rim of the disk. We may then simply take:

$$
\Gamma[g]=\int_{X_{2 n} \times D} \frac{d w}{w} \wedge \operatorname{Tr} \bar{\ell}(\partial \bar{\ell})^{n}
$$

where $\bar{\ell}=g^{-1} \bar{\partial} g$. The action is independent of small changes of extension and a variation of $g$ leads to the equations of motion (7.1). Moreover, $\Gamma[g]$ differs from $\int_{X_{2 n} \times I} \operatorname{Tr}\left(g^{-1} d g\right)^{2 n+1}$ by a local functional of $g$ and hence $\exp \kappa \Gamma[g]$ is single-valued for an appropriate constant $\kappa$.

One can write the action (77.2) in many ways. One way to make contact with the standard WZ term is to take an extension $g(x, w)$ which only depends on the radial coordinates: $g(x, w)=g(x,|w|)$. The integral over the disk reduces to an integral over an interval. This presentation also makes contact with higher-dimensional Chern-Simons theories [14][30]. In four dimensions

$$
\Gamma[g]=\int_{X_{4}} \operatorname{Tr}\left[2(\ell \bar{\ell}-\bar{\ell} \ell) \bar{\partial} \ell+(\bar{\ell} \ell)^{2}\right]+\frac{2}{5} \int_{X_{4} \times I} \operatorname{Tr}\left(g^{-1} d g\right)^{5}
$$

where $\ell=g^{-1} \partial g, \bar{\ell}=g^{-1} \bar{\partial} g$. In the abelian case the chiral cocycle Lagrangian simplifies to

$$
4 \int_{X_{4}} \partial \phi \bar{\partial} \phi \partial \bar{\partial} \phi
$$

for $g=\exp \phi$. This is closely related to the Plebanski action for self-dual gravity [31]. [1]

9 While this equation is rather peculiar it is worth noting that it is not more than second derivatives in any one coordinate and hence probably has a reasonable initial value problem.

10 Quantization of this action and of similar actions has been studied by A. Gerasimov. 
These actions satisfy some curious properties. For example, a four-dimensional extension of the Polyakov-Wiegmann formula is

$$
\begin{aligned}
\Gamma\left[g_{1} g_{2}\right] & =\Gamma\left[g_{1}\right]+\Gamma\left[g_{2}\right] \\
& -2 \int_{X_{4}} \operatorname{Tr}\left[\ell_{1}\left(\bar{r}_{2} \partial \bar{r}_{2}+\partial \bar{r}_{2} \bar{r}_{2}\right)+\bar{r}_{2}\left(\ell_{1} \bar{\partial} \ell_{1}+\bar{\partial} \ell_{1} \ell_{1}\right)-3 \bar{r}_{2} \ell_{1} \bar{r}_{2} \ell_{1}\right]
\end{aligned}
$$

where $r=\partial g g^{-1}, \bar{r}=\bar{\partial} g g^{-1}$. This equation might be of use in trying to define a quantum version of the theory.

\subsection{Nontrivial background metric}

It is instructive to compute a nonchiral partition function $\mathcal{Z}_{\text {nonchiral }}[a, \bar{a}, \gamma]$ in the presence of a nontrivial background Kähler metric $\gamma$. This metric enters the partition function through the measure. (Here we work at fixed complex structure.) As explained in sections five and six, the variation of $\mathcal{Z}_{\text {nonchiral }}[a, \bar{a}, \gamma]$ can be extracted from holomorphic descent. Therefore, in four dimensions we start with the differential form following from the characteristic class:

$$
\begin{aligned}
\left.\operatorname{Td}\left(T^{1,0} X_{4}\right) \operatorname{ch}(E)\right|_{6} & =\frac{1}{3 !} \operatorname{Tr}\left(\frac{i F}{2 \pi}\right)^{3}+\frac{1}{2 !} \operatorname{Tr}\left(\frac{i F}{2 \pi}\right)^{2} \frac{1}{2} c_{1}(R) \\
& +\operatorname{Tr}\left(\frac{i F}{2 \pi}\right)\left[\frac{1}{8}\left(c_{1}(R)\right)^{2}-\frac{1}{24} \operatorname{Tr}\left(\frac{i R}{2 \pi}\right)^{2}\right] \\
& +\frac{r}{48} c_{1}(R)\left[c_{1}(R)^{2}-\operatorname{Tr}\left(\frac{i R}{2 \pi}\right)^{2}\right]
\end{aligned}
$$

Here the curvature on $T^{1,0} X$ is $R=\bar{\partial}\left(\gamma^{-1} \partial \gamma\right)$ and we write $c_{1}(R)=\frac{i}{2 \pi} \bar{\partial} \partial \log \operatorname{det} \gamma \equiv$

$\frac{i}{2 \pi} \bar{\partial} \partial \sigma=\operatorname{Tr}\left(\frac{i R}{2 \pi}\right)$. The trace of powers of $F$ is taken in the representation of $G$, which corresponds to the choice of $E$. In the subsequent formulae this is implicitly understood.

\subsubsection{Nonchiral partition function}

We now use (7.6) to split holomorphically the nonchiral partition function on $\mathcal{A}^{1,1}$ :

$$
\log \mathcal{Z}_{\text {nonchiral }}[a, \bar{a}, \gamma]=\Gamma\left[g_{L}, \gamma\right]+\bar{\Gamma}\left[g_{R}, \gamma\right]+\tilde{\beta}(a, \bar{a}, \gamma)
$$

where $g_{L}, g_{R}$ are defined in (6.4). The chiral splitting function now becomes

$$
\tilde{\beta}=\frac{1}{2 !}\left(\frac{i}{2 \pi}\right)^{2} \beta(a, \bar{a})+\frac{1}{2 !}\left(\frac{i}{2 \pi}\right)^{2} \int \operatorname{Tr}(a \bar{a}) c_{1}(R)
$$


Hence we find:

$$
\Gamma[g ; \gamma]=\Gamma[g]+S_{c_{1}(R)}[g]+\int_{X_{4}}\left[\frac{1}{4}(\operatorname{Tr} R)^{2}-\frac{1}{12} \operatorname{Tr} R^{2}\right] \log \operatorname{det} g
$$

where for any two-form $\Omega$ we define:

$$
S_{\Omega}[g] \equiv-\frac{i}{4 \pi} \int_{X_{4}} \Omega \wedge \operatorname{Tr}\left(g^{-1} \partial g \wedge g^{-1} \bar{\partial} g\right)+\frac{i}{12 \pi} \int_{X_{5}} \Omega \wedge \operatorname{Tr}\left(g^{-1} d g\right)^{3},
$$

and $\log \operatorname{det} g$ in (7.9) is to be understood as $\operatorname{Tr}_{E} \log g$. When $\Omega$ is the Kahler form of a Kahler metric (7.10) is simply the " $W Z W_{4}$ action" studied in $\mathbb{1}$.

\subsubsection{On chiral partition functions}

Equation (7.9) gives $\log \mathcal{Z}_{c h}$ as a function of $\bar{a}$ in a background Kähler metric $\gamma$. The introduction of a nontrivial metric brings up the rather subtle issue of when cocycles should be regarded as trivial or not. A closely related and equally subtle point is the possibility of a $Q$-anomaly in a background Kahler field. By formal $Q$-symmetry the second and third terms in (7.9) should be absent - the dependence of $\mathcal{Z}_{c h}$ on the Kahler metric is $Q$-exact. Nevertheless, the cocycles associated with these terms appear to be nontrivial. In fact, however, if $c_{1}(R)^{2}+c_{2}(R)$ can be written as $\partial \bar{\partial} \alpha_{1}$ (as is always true locally) then we may integrate by parts in the third term to obtain the local counterterm

$$
\delta^{1,0} \int \alpha_{1} \operatorname{Tr} \partial \bar{a}=\int \partial \bar{\partial} \alpha_{1} \log \operatorname{det} g
$$

By the same token, holomorphic factorization cannot determine this term uniquely. There is always the possibility of adding a term $\int \partial \bar{\partial} \alpha \log \operatorname{det} g$ to the action $\Gamma[g]$.

In an similar way, the second term in (7.9) may be regarded as following from a "trivial" cocycle due to the identity:

$$
\delta^{1,0} \int \partial \alpha_{2} \operatorname{Tr}(\bar{a} \partial \bar{a})=2 \int \bar{\partial} \partial \alpha_{2} \operatorname{Tr}(\epsilon \partial \bar{a})
$$

Clearly, one can add $\delta^{1,0}$-exact terms, local in $\bar{a}$ and the external metric $\gamma$ to the chiral cocycle Lagrangian. This point, which plays a role in making contact with the $\mathcal{N}_{w s}=2$ string needs further clarification. 
7.2.3.Dependence of the nonchiral partition function on the background Kähler metric

If we wish to regard the Kähler metric as dynamical then we must include the contribution to the Bott-Chern class coming from the last term in (7.6). These are not determined by holomorphic splitting in $a, \bar{a}$, but are determined from the family index theorem computations. Thus, if we compare the nonchiral partition function in a background Kähler metric $\gamma_{i \bar{j}} d z^{i} d \bar{z}^{\bar{j}}$ to the partition function on a flat background we find [21]:

$$
\Gamma^{\mathrm{conf}}[\gamma]=\frac{1}{96} \frac{1}{(2 \pi)^{2}} \int\left[\partial \sigma \bar{\partial} \sigma(\bar{\partial} \partial \sigma)+\bar{\partial} \sigma \operatorname{Tr}\left(\gamma^{-1} \partial \gamma\right)\left(\bar{\partial}\left(\gamma^{-1} \partial \gamma\right)\right)\right]
$$

where $\sigma=\log \operatorname{det} \gamma$. This is the higher-dimensional analog of the conformal anomaly. Again, the metric dependence comes from the measure of the non-chiral system, as is confirmed by the locality of (7.13). The higher dimensional analogue of the Polyakov action is obtained by considering the difference:

$$
\Gamma^{\operatorname{conf}}[\gamma]-\Gamma^{\operatorname{conf}}[\hat{\gamma}]
$$

For example, $\int \partial \sigma \bar{\partial} \sigma \partial \bar{\partial} \sigma$ is promoted to

$$
\frac{1}{96} \int\left[\frac{1}{4 \pi^{2}} \partial \sigma \bar{\partial} \sigma \partial \bar{\partial} \sigma+\frac{3}{2 \pi i} c_{1}(\hat{R}) \partial \sigma \bar{\partial} \sigma-3\left(c_{1}(\hat{R})\right)^{2} \sigma\right]
$$

where $\sigma=\log \operatorname{det} \gamma-\log \operatorname{det} \hat{\gamma}$.

\section{Diffeomorphism Cocycles}

As a step towards making the complex structure dynamical we now state some results showing how considerations parallel to those in the gauge theory apply to the group of chiral diffeomorphisms. Such diffeomorphisms are defined as follows. Let $f$ stands for a vector of functions $f^{i}\left(z_{1}, \ldots, z_{n}, \bar{z}_{1}, \ldots \bar{z}_{n}\right)$. We consider the $z, \bar{z}$ to be independent 19 and consider the group action: $f \circ g(z, \bar{z}) \equiv f(g(z, \bar{z}), \bar{z})$. We will adopt the active viewpoint: all functions whose arguments are not explicitly indicated are evaluated at $(z, \bar{z})$. We define $J(f)_{i}{ }^{j} \equiv \partial_{i} f^{j}$ and $\bar{J}(f)_{\bar{i}}{ }^{j} \equiv \bar{\partial}_{\bar{i}} f^{j}$. Dot $\cdot$ always denotes (finite dimensional) matrix multiplication.

11 For example, we could work in a real space of signature $(n, n)$. 
We define the Beltrami differential $\bar{\mu}[f]$ to be the matrix:

$$
\bar{\mu}[f]_{\bar{i}}^{j} \equiv\left[\bar{J}(f) \cdot J(f)^{-1}\right]_{\bar{i}}^{j}
$$

One easily verifies the composition law:

$$
\bar{\mu}\left[f_{1} \circ f_{2}\right]=\bar{\mu}\left[f_{2}\right]+\left(\bar{\mu}\left[f_{1}\right] \circ f_{2}\right) \cdot J\left(f_{2}\right)^{-1}
$$

and hence, under the infinitesimal right action $f \rightarrow f \circ \ell$ defined by $\ell=z-v(z, \bar{z})$ we have right action on the space of Beltrami differentials:

$$
\bar{\mu}\left[f_{1}\right] \rightarrow \bar{\mu}\left[f_{1}\right]-\bar{\partial} v-v^{i} \partial_{i} \bar{\mu}\left[f_{1}\right]+\bar{\mu}\left[f_{1}\right] \partial v \quad .
$$

Here $\partial v=\partial_{i} v^{j}$ is regarded as a matrix-valued function.

Let us now try to find an action $\Gamma[f]$ whose variation under the right chiral diffeomorphism is a local functional of the Beltrami differential. Such an action must be the result for the $\bar{\mu}$-dependence of the chiral partition function (1.2). The answer is easily motivated by recalling the close relation between the diffeomorphism and local Lorentz anomalies.

We define the action for diffeomorphisms to be:

$$
\Gamma[f] \equiv \Gamma\left[J(F)^{-1}\right]
$$

where, to any $f$ we associate the inverse, denoted as $F$ and defined by $f \circ F=F \circ f=z$ and where on the right hand side we use the action (1.9) for a $G L(n, \mathbb{C})$ matrix. Under the right diffeomorphism action $f \rightarrow f \circ \ell, \ell=z-v(z, \bar{z})$, which is equivalent to $\bar{\mu} \rightarrow \delta_{v} \bar{\mu}$ we have

$$
\delta_{v} \Gamma[f]=-12 \int_{X_{4}} \operatorname{Tr}\left[(\partial v)\left(\mathcal{R}_{\bar{\mu}}\right)^{2}\right]
$$

where we define a $(1,1)$-form $\mathcal{R}_{\bar{\mu}}$ with values in $g l(n, \mathbb{C})$ by

$$
\left(\mathcal{R}_{\bar{\mu}}\right)_{m}^{p} \equiv d z^{j} d \bar{z}^{\bar{k}}\left(\partial_{j} \partial_{m} \bar{\mu}_{\bar{k}}^{p}\right)
$$

Comparing with (5.18) we see that we have produced the group cocycle for diffeomorphisms.

This action defines higher dimensional generalizations of the Virasoro algebra. Indeed, since there are many invariant polynomials on the Lie algebra $g l(n, \mathbb{C})$ there are many generalizations of the Virasoro algebra corresponding to different representations of $g l(n, \mathbb{C})$ in (8.5). For example, to get the cocycle related to the Todd class in 4 dimensions one must choose the virtual representation $R$ of $U(2)$ such that $\operatorname{Tr}_{R}(x)=\frac{1}{48}\left[\left(\operatorname{Tr}_{F}(x)\right)^{3}-\right.$ $\operatorname{Tr}_{F}(x) \operatorname{Tr}_{F}\left(x^{2}\right)$ ], where $F$ is the fundamental of $U(2)$. This concludes the computation of the $\bar{\mu}$ dependence of the chiral partition function for four-dimensions. 


\section{Hypermultiplets and self-dual geometry}

In this section we restrict attention to four dimensions. For $b c$ in a general representation of the gauge group the equation (7.1) is fourth-order in derivatives. However, there is one very special class of representations leading to more conventional field theories. Let us choose a vector bundle of the form $\left(E \oplus E^{*}\right) \otimes H$ where $H$ is a line bundle. In more physical terms, we consider a gauge group $U(N) \times U(1)$ with left-handed fermions in the representation

$$
(\rho,+1) \oplus\left(\rho^{*},+1\right)
$$

Given the relation of $b c$ systems to twisted supersymmetry explained above we see that the representation (9.1) is extremely natural yet special: it is simply a hypermultiplet for an $\mathcal{N}=2 U(N)$ supersymmetric gauge theory.

Note that the choice of representation leads to a cancellation of the standard nonabelian $\left(g^{-1} d g\right)^{5}$ term in the group cocycle. Denoting the $U(N) \times U(1)$ gauge field by $\left(A, A_{a b}\right)$ we have, in an $F^{0,2}=0$ background $A^{0,1}=g^{-1} \bar{\partial} g, A_{a b}^{0,1}=\bar{\partial} \varphi$. The action (7.3) evaluated for the representation (9.1) is:

$$
\log \mathcal{Z}_{c h}=S_{\Omega}(g)+\frac{1}{3 !} \frac{1}{(2 \pi i)^{2}} \int \varphi(\partial \bar{\partial} \varphi)^{2}+\int \varphi \frac{r}{12}\left(c_{1}(R)^{2}+c_{2}(R)\right)
$$

The first term is the $W Z W_{4}$ action defined in (7.10) above and evaluated now for $\Omega=$

$\frac{1}{2 \pi i} \partial \bar{\partial} \varphi$. The equations of motion (considering the metric to be nondynamical) following from (9.2) are:

$$
\begin{aligned}
-\frac{4 \pi^{2} r}{12}\left(c_{1}(R)^{2}+c_{2}(R)\right)+\frac{1}{2} \operatorname{Tr}\left(\bar{\partial}\left(g^{-1} \partial g\right)\right)^{2}+\frac{1}{2} \partial \bar{\partial} \varphi \wedge \partial \bar{\partial} \varphi & =0 \\
(\partial \bar{\partial} \varphi) \wedge \bar{\partial}\left(g^{-1} \partial g\right) & =0
\end{aligned}
$$

An immediate corrollary of this result is that we have fermionized the algebraic sector current correlators described in [1], in analogy to fermion/boson correspondence in $W Z W_{2}$. The connection between (9.1) and the $W Z W_{4}$ theory was pointed out in [1]. It has also been discussed recently in [32]. 


\subsection{Comparison with the $\mathcal{N}_{w s}=2$ string}

The spacetime physics of the $\mathcal{N}_{w s}=2$ string has been related to self-dual geometry in [33] [31]. The field content of the open $\mathcal{N}_{w s}=2$ string is given by a scalar field $K$ and adjoint-valued fields $\pi^{a}(x)$. The equations of motion are [33]:

$$
\begin{aligned}
\partial \bar{\partial} K \wedge\left(\bar{\partial} g^{-1} \partial g\right) & =0 \\
\partial \bar{\partial} K \wedge \partial \bar{\partial} K & =\omega_{0}^{2}+\operatorname{ch}_{2}(F)
\end{aligned}
$$

Comparing with (9.3) we see that identifying $K$ with $\varphi$ and $g$ with $e^{\pi}$ we obtain similar equations. According to the remarks in section 7.2.2 above we can introduce local counterterms of the type $(\overline{7.11})(\sqrt{7.12})$ to bring (9.3) to precisely the form (9.4) of the open $\mathcal{N}_{w s}=2$ string.

\section{Conclusions}

In conclusion we comment on a few possible applications of the above results.

The next step following the above investigation is the formulation of the higher dimensional analogs of the Knizhnik-Zamolodchikov equations. This is currently under study. Moreover, the relation to the $W Z W_{4}$ theory opens the way to an investigation of some representations of higher dimensional current algebras. Holomorphic gauge transformations lead to $Q$-exact changes in the action. We may thus expect that the $Q$-cohomology of the free field Hilbert space of a Kähler-twisted chiral supermultiplet (more generally of a $b c$ system on any complex manifold) supports a representation of higher loop gauge algebras. We hope to discuss this elsewhere [34]. It is quite likely that representations related to $b c$ systems will shed some light on the results of Borcherds and Jorgenson and Todorov concerning determinants of $\bar{\partial}$ operators on Calabi-Yau manifolds 35] 36].

An important avenue for generalizing these results is the generalization from Kähler to Hermitian metrics. This can probably be accomplished using the supersymmetric quantum mechanics techniques discussed above. This generalization is necessary to discuss the relation of the diffeomorphism anomalies with the "higher dimensional conformal anomalies" such as (7.13). This is currently under investigation [37.

There are other interesting generalizations defined, for example adding superpotentials, dynamical vectormultiplets, and supergravity. It remains to be seen if these are tractable. Some results in this direction have been obtained in [11]. 
Also, given the close analogy to [7] [8], it is natural to expect that these results will have an application in defining a four-dimensional analog of bosonization.

Finally, these results might find some application in understanding string duality. First of all, the diffeomorphism cocycles we have uncovered might be of some use in quantization of $p$-branes as quantum mechanical objects. Second, the $\mathcal{N}_{w s}=(2,1)$ heterotic string has recently played a role in attempts to achieve a deeper understanding of string duality [38]. The relevant geometry appears to be self-dual geometry with torsion. It would be very interesting to generalize the results of this letter to include these systems.

\section{Acknowledgements}

We are grateful to Anton Gerasimov for many comments related to the issues discussed in this paper and for sharing his insights with us. N.Nekrasov wishes to thank the Erwin Schrödinger International Institute for Mathematical Physics at Vienna where some of the ideas of this paper have appeared and especially K.Gawedzki and H.Grosse for hospitality. He is also grateful to A. Rosly and V. Fock for useful discussions. S. Shatashvili would like to thank the CERN theory group for hospitality during the completion of this paper. The research of A. Losev was partially supported by RFFI grant 96-01-01101 and Volkswagen Stiftung. The research of G. Moore is supported by DOE grant DE-FG02-92ER40704, and by a Presidential Young Investigator Award. The research of N. Nekrasov is supported by the Porter Ogden Jacobus fellowship. The research of S. Shatashvili is supported by DOE grant DE-FG02-92ER40704, NSF CAREER award,

DOE OJI award, and the Alfred P. Sloane Foundation. 


\section{References}

[1] A. Losev, G. Moore, N. Nekrasov, S. Shatashvili, "Four-Dimensional Avatars of 2D RCFT," hep-th/9509151.

[2] A. Losev, G. Moore, N. Nekrasov, S. Shatashvili, "Central Extensions of Gauge Groups Revisited," hep-th/9511185.

[3] Further results appear in N. Nekrasov, PhD Thesis

[4] E. Witten, "Nonabelian bosonization in two dimensions," Commun. Math. Phys. 92 (1984) 455

[5] A. M. Polyakov, "Gauge transformations and diffeomorphisms," Int. J. Mod. Phys. A5: 833, 1990;

"Quantum gravity in two dimensions," Mod. Phys. Lett. A2: 893,1987; "Quantum geometry of bosonic strings," Phys. Lett. 103B (1981) 207-210

[6] V.P. Nair and Jeremy Schiff, "Kahler Chern Simons theory and symmetries of antiselfdual equations" Nucl.Phys.B371:329-352,1992; "A Kahler Chern-Simons theory and quantization of the moduli of antiselfdual instantons," Phys.Lett.B246:423-429,1990, "Topological gauge theory and twistors," Phys.Lett.B233:343,1989

[7] A. M. Polyakov and P. B. Wiegmann, Phys. Lett. B131(1983)121

[8] A. M. Polyakov, "Two dimensional quantum gravity: superconductivity at high $T_{c}$ ", Les Houches lectures, In *Les Houches 1988, Proceedings, Fields, strings and critical phenomena* $305-368$

[9] D. Friedan, E. Martinec, and S. Shenker, "Conformal Invariance, Supersymmetry, and String Theory," Nucl.Phys. B271 (1986) 93.

[10] V.G. Knizhnik, "Analytic fields on Riemann surfaces," In *Nishinomiya 1987, Proceedings, Quantum string theory* 120-131.

[11] A. Johansen, "Twisting of $N=1$ susy gauge theories and heterotic topological theories," Int.J.Mod.Phys.A10:4325-4358,1995, hep-th/9403017;

"Infinite Conformal Algebras in Supersymmetric Theories on Four Manifolds," Nucl.Phys.B436:291-341,1995, hep-th/9407109;

"Realization of $W_{1+\infty}$ and Virasoro algebras in supersymmetric theories on four manifolds," Mod.Phys.Lett.A9:2611-2622,1994, hep-th/9406156

[12] J.-S. Park, "Holomorphic Yang-Mills theory on compact Kahler manifolds," hepth/9305095; Nucl. Phys. B423 (1994) 559;

J.-S. Park, " $N=2$ Topological Yang-Mills Theory on Compact Kähler Surfaces", Commun. Math, Phys. 163 (1994) 113;

J.-S. Park, " $N=2$ Topological Yang-Mills Theories and Donaldson Polynomials", hep-th/9404009

[13] E. Witten, "Supersymmetric Yang-Mills Theory On A Four-Manifold," J. Math. Phys. 35 (1994) 5101. 
[14] L. Faddeev and S. Shatashvili, Theor. Math. Fiz., 60 (1984)206;

L. Faddeev, Phys. Lett. B145 (1984) 81.;

J. Mickelsson, Commun. Math. Phys. , 97 (1985) 361.

[15] B. Zumino, "Chiral anomalies and differential geometry," in Relativity, Groups and Topology II, proceedings of the Les Houches summer school, Bryce S DeWitt and Raymond Stora, eds. North-Holland, 1984.

[16] For reviews see Symposium on Anomalies, Geometry and Topology William A. Bardeen and Alan R. White, eds. World Scientific, 1985, and L. Alvarez-Gaumé and P. Ginsparg, "The structure of gauge and gravitational anomalies," Ann. Phys. 161 (1985) 423; L. Alvarez-Gaumé, "An introduction to anomalies," Lectures given at Int. School on Mathematical Physics, Erice, Italy, Jul 1-14, 1985. Published in Erice School Math.Phys.1985:93

[17] W. A. Bardeen, "Self-Dual Yang-Mills, Integrability and Multi-Parton Amplitudes", Fermilab - Conf - -95-379-T, Aug 1995, Presented at Yukawa International Seminar '95: 'From the Standard Model to Grand Unified Theories', Kyoto, Japan, 21-25 Aug 1995.

[18] D. Cangemi, "Self-dual Yang-Mills Theory and One-Loop Like-Helicity QCD MultiGluon Amplitudes," hep-th/9605208.

[19] T. Banks, "Vertex Operators in 2D Dimensions," hep-th/9503145

[20] N. Berline, E. Getzler, and M. Vergne, Heat Kernels and Dirac Operators Springer

[21] J. -M. Bismut, H. Gillet, and C. Soulé, "Analytic Torsion and Holomorphic Determinant Bundles, I.II.III" CMP 115(1988)49-78;79-126;301-351

[22] M.F. Atiyah and I.M. Singer, "Dirac operators coupled to vector bundles," Proc. Natl. Acad. Sci. 81 (1984) 2597

[23] This interpretation has been widely discussed in the physics literature. For examples, see: L. Alvarez-Gaumé and P. Ginsparg, "The structure of gauge and gravitational anomalies," Ann. Phys. 161 (1985)423;

G. Moore and P. Nelson, "The Aeteology of Sigma Model Anomalies," Comm. Math. Phys. 100(1985)83.

[24] S. Donaldson, "Anti-Self-Dual Yang-Mills Connections over Complex Algebraic Surfaces and Stable Vector Bundles," Prod. Lond. Math. Soc. 50(1985)1.

[25] S. Donaldson, "Infinite Determinants, Stable Bundles, and Curvature," Duke Math. J. , 54 (1987) 231.

[26] L. Alvarez-Gaumé, "Supersymmetry and Atyah-Singer index theorem", Commun.Math.Phys.90:161,1983

[27] D. Friedan and P. Windey, "Supersymmetric derivation of the Atyah-Singer index and the chiral anomaly," Nucl.Phys.B235:395,1984

[28] L. Alvarez-Gaumé and E. Witten, "Gravitational Anomalies," Nucl. Phys. B234(1983) 269 
[29] R. Bott and S.S. Chern, "Hermitian vector bundles and the equidistribution of the zeroes of their holomorphic sections," Act. Math. 114(1965)71

[30] V. Fock, N. Nekrasov, A. Rosly, and K. Selivanov, "What we think about higherdimensional Chern-Simons theories," Published in Sakharov Conf.1991:465-472

[31] H. Ooguri and C. Vafa, "Self-Duality and $N=2$ String Magic," Mod.Phys.Lett. A5 (1990) 1389-1398;

"Geometry of $N=2$ Strings," Nucl.Phys. B361 (1991) 469-518;

" $N=2$ Heterotic Strings, Nucl.Phys.B367:83-104,1991

[32] Maximo Banados , Luis J. Garay , Marc Henneaux, "The dynamical structure of higher dimensional Chern-Simons theory," hep-th/9605159

[33] N. Marcus, "A tour through $N=2$ strings," hep-th/9211059

[34] A. Losev, G. Moore, N. Nekrasov, S. Shatashvili, "Supersymmetry and the representations of four-dimensional current groups," in progress.

[35] R. Borcherds, "The moduli space of Enriques surfaces and the fake monster Lie superalgebra," preprint (1994).

[36] J. Jorgenson and A. Todorov, "A conjectured analog of Dedekind's eta function for K3 surfaces," Math. Res. Lett. 2(1995) 359; "Enriques surfaces, Analytic discriminants, and Borcherd's $\Phi$ function," Yale preprint.

[37] A. Losev, G. Moore, N. Nekrasov, S. Shatashvili, in progress.

[38] D. Kutasov, E. Martinec, "New Principles for String/Membrane Unification." hepth/9602049;

D. Kutasov , E. Martinec, M. O' Loughlin, "Vacua of M-theory and N=2 strings," hep-th/9603116 